\title{
Resilience of Error Correction Codes Against Harsh Electromagnetic Disturbances: Fault Elimination for Triplication-based Error Correction Codes
}

\author{
Jonas Van Waes, Student Member, IEEE, Dries Vanoost, Jens Vankeirsbilck, Student Member, IEEE, \\ Jonas Lannoo, Davy Pissoort, Senior Member, IEEE, Jeroen Boydens, Member, IEEE
}

\begin{abstract}
Modern safety-critical systems rely heavily on robust communication channels. Even though these communication channels can be protected by Error Detection and Correction Codes, vulnerabilities caused by False Negatives still exist. These False Negatives can be caused by harsh electromagnetic disruptions and are detriment to overall safety. This paper considers the construction and structure of Triplication-based Error Correction Codes to find the most EMI-resilient code. Each code is tested and simulated in terms of the occurrence rate of False Negatives under single-frequency disturbances. It is found that a code with inversion is significantly more robust to these disturbances. Furthermore, a systematic fault injection is also performed to search for vulnerabilities within the code itself. The systematic approach allows us to reverse-engineer the expected vulnerabilities to real-world disturbances.
\end{abstract}

Index Terms-Electromagnetic Interference, Communication Channel, Error Correction and Detection Codes, Reverberation Room Simulation

\section{INTRODUCTION}

A LREADY for many years, we are seeing a constant increase in the use of Electrical, Electronic and Programmable Electronic (E/E/PE) devices in our everyday lives. Over the last decade, these E/E/PE devices are performing more and more safety-critical tasks, whereby failure of the device might lead to harm to humans, the environment, or bear significant financial costs [1]. This trend is expected to continue due to the emergence of applications like, amongst others, autonomous vehicles, autonomous airplanes, autonomous vessels, highly-automated factories and care robots.

Many of these safety-critical applications are, or will be, highly dependent on (wireless) communications. Disruptions of those communications by Electromagnetic Interference (EMI) might render these devices inactive or unavailable. Even worse, specific disruptions might lead to dangerous failures of the devices. Whether the disruptions occur by accident (unintentional EMI) or maliciously (intentional EMI), it is

Manuscript received June 13, 2019; revised September 02, 2019.

This work is supported by a research grant from the Flemish Agency for Innovation and Entrepreneurship (VLAIO) within the CORNET/TETRA RESSIAR-MID/TransSIMS (HBC.2017.0650) project.

J. Van Waes, J. Vankeirsbilck and J. Boydens are with the Department of Computer Science, KU Leuven Campus Bruges, Spoorwegstraat 12, 8200 Bruges, Belgium (e-mail: jonas.vanwaes@kuleuven.be; jens.vankeirsbilck@kuleuven.be; jeroen.boydens@kuleuven.be)

J. Lannoo, D. Vanoost and D. Pissoort are with the Department of Electrical Engineering, KU Leuven Campus Bruges, Spoorwegstraat 12, 8200 Bruges, Belgium (e-mail: dries.vanoost@kuleuven.be; jonas.lannoo@kuleuven.be; davy.pissoort@kuleuven.be important to limit the influences of these disturbances. The electromagnetic working environment of a device is suspected to become even harsher and denser, partly by the outrol of $5 \mathrm{G}$ networks.

The protection against EMI has always been the domain of Electromagnetic Compatibility (EMC Engineering), while limiting and reducing the safety-related risks associated with a system has been the responsibility of Functional Safety (FS Engineering). Unfortunately, these two engineering disciplines have evolved independently, leading to different terminologies and techniques [2], [3]. Nevertheless, it is essential that these two domains converge as much as possible in order to limit the influence of, and safety-related risks induced by, Electromagnetic Interference.

In [4], a selection of Error Detection and Correction Codes (EDCCs) - which are frequently used in FS Engineering have been studied and compared in the context of harsh EMI. That research determined the fault mechanisms associated with continuous-wave electromagnetic disturbances. More specifically, it was studied in depth why and under which conditions EDCCs can generate False Negatives. False Negatives arise when corrupted data is deemed valid at the receiving side. The system does not know the data was corrupted and cannot take any countermeasures. Hence, these kind of failures are detrimental to the overall safety.

Within this paper, the insights of [4] are used to cope with False Negatives under reverberation room conditions. From the state of the art mentioned in [4], this paper proposes optimized EDCCs that completely eliminate the occurrence of False Negatives under continuous wave disturbances.

The remainder of this paper is organized as follows. Section II details the experimental setup. To enable an effective comparison, the most EMI-resilient EDCC from [4] (simple triplication) is selected and used as a baseline for further experiments. Details about this baseline are given in Section III. In Section IV, the effects on the resilience of the codes are tested via a Monte-Carlo-based EMI-simulation framework. To explore the performance of EM-diverse techniques, the baseline and deducted codes are tested with a systematic fault injection model in Section V. Finally, conclusions are drawn in Section VI.

\section{EXPERIMENTAL SETUP}

To assist the reader in his/her understanding of the remainder of this paper, we repeat Section II of [4]. Moreover, 


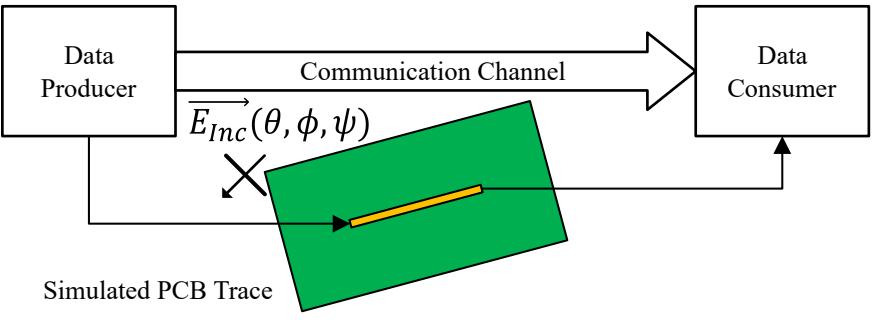

Fig. 1. Simulated Trace as Communication Channel [6]

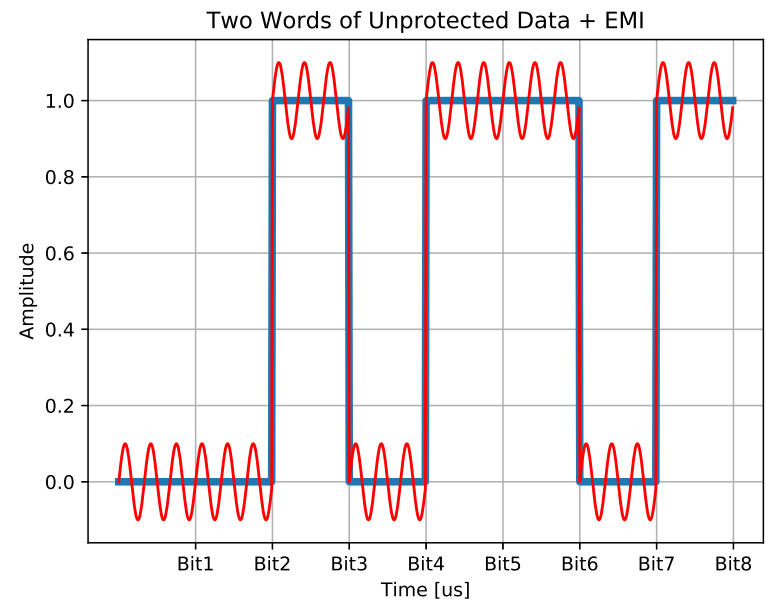

Fig. 2. The considered signals in the time domain. The blue line represents a transmitted bit stream. The red line is the received signal: the induced disturbance voltage added to the transmitted bit stream.

Section II-A is a repetition from Section III of [5] where the induced voltage calculation is elaborated.

Fig. 1 gives a conceptual overview of the experimental setup that will be considered in the remainder of this paper. This setup was previously used in [5] and [6]. The Device Under Test (DUT) comprises a Printed Circuit Board (PCB) with a single $50 \Omega$ micro-strip. One end of the micro-strip is driven with a $1 \mathrm{~V}$ power supply with a $50 \Omega$ output impedance. The other end of the micro-strip is terminated with a $50 \Omega$ load. The PCB itself is an FR4 substrate of $10 \mathrm{~cm}$ by $16 \mathrm{~cm}$ and has a thickness of $1.6 \mathrm{~mm}$, with a dielectric constant of 4.7 . The trace is $3 \mathrm{~mm}$ wide and has a length of $5 \mathrm{~cm}$. The bottom of the PCB is covered with a ground plane.

A Data Producer generates data words, encodes them (either with or without an EDCC) and puts these code words onto the communication channel (the PCB trace in this case). The channel then transmits the code words to the Data Consumer. The latter decodes the received code words to data words. The entire communication subsystem is subjected to an EMI disturbance, represented schematically in Fig. 1 by $E_{\text {inc }}$. In order to check the effectiveness of the considered EDCCs, the decoded data word is compared to the original data word that was sent. A conceptual overview of the considered time signals can be seen in Fig. 2.

For this paper, the electromagnetic disruption under consideration is a single-frequency disturbance. The simulations themselves easily implement a multi-frequency disturbance.

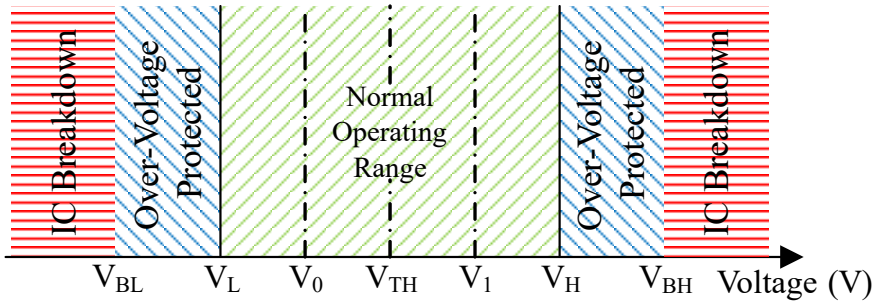

Fig. 3. Decoding Voltage Thresholds (Section II-B)

However, this complicates the illustrative potential of the figures within this paper.

\section{A. Calculation of the Induced Voltages}

Calculating the induced voltages at the end of the microstrip due to incoming disturbances can be done using several methods. One of those methods is using a full-wave simulation for each possible way an EMI disturbance could disturb the DUT. This would require a huge amount of simulations and simulation time. Therefore, an efficient reciprocity-based algorithm is used. This method uses only one full-wave simulation per port. In each of these simulations, one port is excited and the far-field of the DUT is calculated. Using the far-field data and the reciprocity theorem, the induced voltages can be calculated on all ports as a post-processing step for whatever incoming EMI disturbance [7].

In addition, this paper considers the harsh EMI-environment to be a reverberating environment, because of its similarities with the real world, comprising a lot of reflections [5]. These conditions are created by using the plane wave integral representation for reverberation chambers as presented in [8]. This states that a reverberating environment can be represented by a superposition of randomly chosen plane waves (according to the appropriate statistical distributions). The amount of plane waves that are combined together could be represented as the amount of modes that are simultaneously excited in a real reverberating environment [9].

The plane waves that are combined are a set of $\mathrm{N}$ plane waves, each with random properties for the polar angle $\theta$, azimuth angle $\varphi$, polarization angle $\psi$ and phase angle $\alpha$. To represent the continuously changing nature of a reverberation environment, this simulation is done $\mathrm{M}$ times and each time a different set of $\mathrm{N}$ plane waves is chosen. For this study, $\mathrm{M}$ and $\mathrm{N}$ were chosen to be 10,000 and 200 , respectively.

\section{B. Over-voltage Protection}

It is reasonable to assume that well-designed safety-critical systems are protected against over-voltages at, for example, the pins of Integrated Circuits (ICs). Therefore, there are several thresholds to be considered when decoding a received voltage to a logic ' 1 ' or ' 0 ' bit value. This is schematically depicted in Fig. 3.

The normal operating voltage range goes from $V_{\mathrm{L}}$ to $V_{\mathrm{H}}$, whereas the over-voltage protection is activated between $V_{\mathrm{BL}}$ to $V_{\mathrm{L}}$ (low end) and between $V_{\mathrm{H}}$ to $V_{\mathrm{BH}}$ (high end). For voltages below $V_{\mathrm{BL}}$ or above $V_{\mathrm{BH}}$, the IC will break down. The 
latter is a situation which must be avoided as much as possible. In a well-designed safety-critical system, IC breakdown is avoided by the over-voltage protection mechanisms. These mechanisms clamp the voltage to a voltage within the blue region on Fig. 3, preferably well below $V_{\mathrm{BH}}$ or above $V_{\mathrm{BL}}$.

In the remainder of this paper, we assume that the system can actually detect and know that the over-voltage protection has been triggered, as described in [10]. This allows the initiation of the necessary steps to ensure overall safety is maintained. This can be the transitioning to a safe state, if such a state exists in the application at hand, or transitioning to a degraded mode in which only limited functionality is available.

For all examples further considered in this paper, a NonReturn to Zero Level encoding is used, as explained in [11] and [12]. Nominal voltages for a logical ' 0 ' and ' 1 ' are $V_{0}=$ $0.0 \mathrm{~V}$ and $V_{1}=0.5 \mathrm{~V}$, respectively. To achieve the maximum noise margin between both nominal values, the bit decoding threshold $V_{\mathrm{TH}}$ was chosen to be half the distance of $V_{0}$ and $V_{1}$. The same noise margin was chosen for both the lower and upper over-voltage detection thresholds: $V_{\mathrm{L}}=-0.25 \mathrm{~V}$ and $V_{\mathrm{H}}=0.75 \mathrm{~V}$. For sampling points below $V_{\mathrm{L}}$ or above $V_{\mathrm{H}}$, the over-voltage detection is triggered. Note that $V_{\mathrm{BL}}$ and $V_{\mathrm{BH}}$ are determined by the characteristics of the used IC.

\section{Fault Categorization}

Following the different voltage thresholds defined in Fig. 3, five distinct categories are considered after decoding. Each category is marked with a distinct color from Fig. 4 onwards:

- Category 1: The decoded data is correct and no correction at all had to be made. This is the normal, expected behavior and is marked in green;

- Category 2: The decoded data is correct, but only after the correction of the applied EDCC. The system knows that the data has been altered by the EDCC and that care must be taken in processing the data (e.g. transitioning to a degraded mode with limited functionality). However, this is still an acceptable category and is marked in yellow;

- Category 3: The decoded data is incorrect, despite an attempt of the EDCC to correct it. The system knows that the data has been altered by the EDCC and that care must be taken in processing the data (e.g. transitioning to a degraded mode with limited functionality). This category is undesired and marked in orange;

- Category 4: The decoded data is incorrect, but the EDCC did not even detect that one or multiple bit errors have occurred. Thus, the system incorrectly assumes that all is ok, which could easily lead to (very) dangerous failures. This scenario is detrimental to the overall system safety and is therefore marked in red. This category is also known as a False Negative;

- Category 5: The over-voltage protection is triggered and a well-designed safety-critical system induces an operation to ensure the overall system safety is maintained. This is a desired category and marked in blue.

An important focus of this paper is to study how the different EDCCs affect the rate of False Negatives indicated with the red category. For safety-critical systems this category is to be avoided because no countermeasures can be taken. The system is unaware that the received data is incorrect which could result in catastrophic failures.

\section{Monte-Carlo-based Simulation Framework}

As stated above, the DUT is subjected to harsh EMI disturbances (being a superposition of a large number of plane waves at the same frequency $f_{\mathrm{EMI}}$ ). As we want to draw general conclusions, a Monte Carlo simulation is performed as presented in [11] and [12]. In this Monte Carlo simulation, the parameters of the incident plane waves are chosen randomly, while also statistically varying the time delay between the disturbance and the transmitted bits. Moreover, in our study, we swept the bit rate (denoted as $B R$ in all formulas and equations) from $1 \mathrm{Mbps}$ to $1500 \mathrm{Mbps}$ in steps of $1 \mathrm{Mbps}$, while $f_{\mathrm{EMI}}$ equalled ten specific values as noted in Eq. 1.

Previous experiments already showed a correlation between the ratio of $f_{\mathrm{EMI}}$ and $B R$ on the one hand, and the susceptibility to EMI on the other hand [6], [11], [12]. Therefore, ten frequencies (from which most are prime frequencies) were selected and the bit rate was swept. The goal was to explicitly uncover the correlation and influence of the bit rate to disturbance frequency ratio. Also, it is unfeasible to perform a full coverage of all possible frequencies, bit rates and incoming field strengths. This continuous state space is too wide to cover. Therefore, a subset has been chosen. Note that the fault injection model, described in Section $\mathrm{V}$ does consider all possible faults. However, no consideration of the actual likelihood of the disturbance occurring in the field have been implemented.

Since similar results were obtained for different incident field strengths and communication channel threshold voltages, the incident field strength is fixed to $400 \mathrm{~V} / \mathrm{m}$, which is a representable value for all examples considered in the remainder of this paper. Since the results of the Monte Carlo simulations are similar for each $f_{\mathrm{EMI}}$, the remainder of this paper only describes the results for $f_{\mathrm{EMI}}=683 \mathrm{MHz}$ (unless explicitly stated otherwise).

$$
\left\{\begin{aligned}
& f_{\mathrm{EMI}}=\{97,100,257,313,419,439, \\
&500,683,797,929,967\} \mathrm{MHz} \\
& B R \in \quad[1,1500] \mathrm{Mbps} \mid \Delta B R=1 \mathrm{Mbps} \\
& E_{\text {inc }}= 400 \mathrm{~V} / \mathrm{m}
\end{aligned}\right.
$$

\section{ChARACTERISTICS OF THE BASEline}

From [4], it can be concluded that a simple triplication method is the most effective in terms of limiting the number of False Negatives under harsh EMI disturbances. The simple triplication method is denoted as TEC, since it sends the data three times (Triplication), without inversion (Equal) and bit by bit (Close together). The encoding scheme for a 4-bit data word is provided in Eq. 2 as reference.

$$
T E C(A B C D)=[A A A B B B C C C D D D]
$$

The 12-bit code word can then be transmitted to the receiver. At the receiver end, the code word is decoded and corrected if 


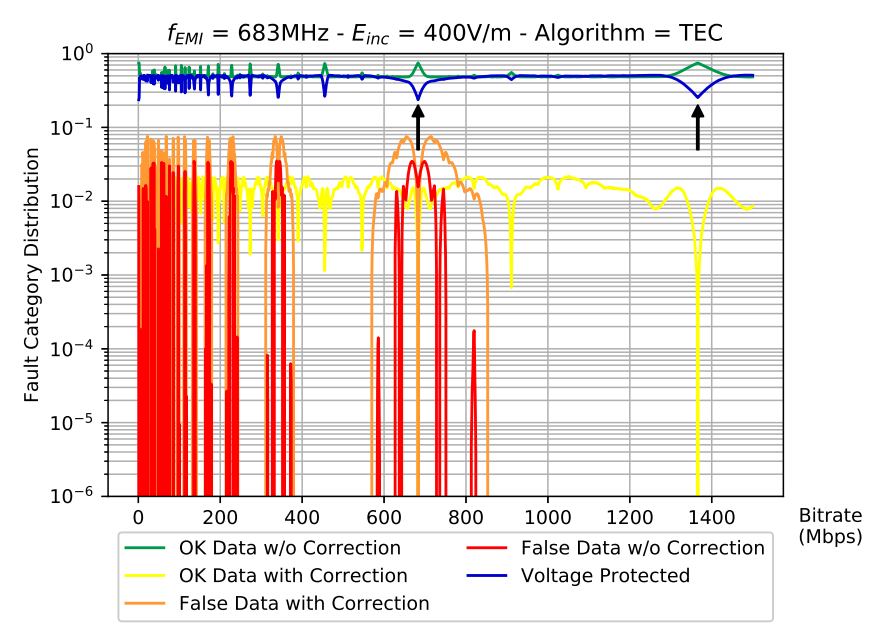

Fig. 4. TEC Fault Categorization

needed, after which the data can be used for further processing. This simple EDCC serves as the starting point and baseline for the study in this paper that aims to implement specific adaptations to TEC in order to gain a significant reduction the occurrence of unwanted False Negatives.

In order to perform effective adaptations, the baseline should be analyzed in full detail and its weaknesses revealed. From [4], specific fault mechanisms were already identified. This section will use those fault mechanisms in a deeper analysis to eliminate False Negatives and to design more EMIresilient EDCCs.

\section{A. Baseline Observations}

The results for the baseline experiment with TEC are depicted in Fig. 4. All categories described in Sec. II-C are present. In order to have a global performance metric, the average of each category is calculated. These averages are the starting point and will serve as a comparison metric to assess the effectiveness of the designed EMI-resilient codes (see Section IV), which are used to reduce the rate of False Negatives.

The over-voltage protected category (blue) has an average value of $46.6 \%$. This means that on average, under the considered EMI disturbances, half of the transmissions of a code word will trigger the over-voltage protection system. This percentage is mainly due to four individual factors. Two of them are system design factors: (1) the length of the considered code word; and (2) the considered nominal bit encoding voltage levels $V_{0}$ and $V_{1}$, and the over-voltage protection system thresholds $V_{\mathrm{L}}$ and $V_{\mathrm{H}}$ (Fig. 3). The other two factors are typical electromagnetic characteristics, i.e. (1) the strength and (2) the frequency of the incoming electromagnetic disturbance.

Fig. 4 also shows that for some specific bit rates, the blue category (voltage protected) decreases significantly. This closely relates to a major increase in the green category, which represents the error-free code words. Both relate very closely to one another and the effect is caused by the specific relation between the bit rate and the disturbance frequency $f_{\mathrm{EMI}}$. The full explanation of these observations can be read in Section III.B of [4] and only a summary is provided here, as it is of great importance for the understanding of the remainder of this paper.

The bit rates for the increased performance (green category/error-free data) can be represented by Eq. 3. These bit rates can be substituted into the equation describing the discrete sampling points $t_{k}$ denoted in Eq. 4 . The result of the substitution is then substituted in the EMI sine wave $v_{\mathrm{EMI}}\left(t_{k}\right)$, defined with $f_{\mathrm{EMI}}$, described by Eq. 5. After all substitutions, the induced voltage on the PCB trace at the sampling points is obtained as illustrated in Eq. 6. The code length $L$ in Eq. 4 is 12 bits for this paper.

$$
\begin{gathered}
B R=\frac{2 f_{\mathrm{EMI}}}{n} \mid n \in \mathbb{N}_{0} \\
t_{k}=\frac{k}{B R}+\frac{1}{2 B R} \mid k \in[0, L-1] \\
v_{\mathrm{EMI}}\left(t_{k}\right)=A \sin \left(2 \pi f_{\mathrm{EMI}} t_{k}+\phi_{\mathrm{EMI}}\right) \\
v_{\mathrm{EMI}}\left(t_{k}\right)=A \sin \left(\frac{\pi n}{2}(2 k-1)+\phi_{\mathrm{EMI}}\right)
\end{gathered}
$$

In Eq. 6, the $n$-value is fixed for a given bit rate. The $k$ values denote the sampling points and range from zero to $L-1$ (i.e. 11 for the examples provided in this paper). Since $n$ is fixed, two specific cases arise, namely one for when $n$ is odd and one for when $n$ is even: the sampled voltages are only dependent on $\cos \left(\phi_{\mathrm{EMI}}\right)$ and $\sin \left(\phi_{\mathrm{EMI}}\right)$, respectively. Thus, for even $n$, the sampling points all have the same value for a logical ' 1 ' or logical ' 0 ', i.e. the EMI-induced voltage plus the nominal value of the encoded bit $\left(V_{0}\right.$ or $\left.V_{1}\right)$. On the other hand, for odd $n$, an alternating pattern ('...010101...') in EMIinduced voltage arises which can cause False Negatives. These observations are depicted in Fig. 5. The combination of these alternating characteristics of the sampled voltages and the code word construction causes the vulnerabilities of the baseline towards False Negatives of the code under EMI conditions. This is explained in more detail in Section III-B.

For the other categories presented in Fig. 4, the following averages are calculated. The yellow category (successful correction) averages $1.44 \%$. The orange category (failed correction) averages out around $1.05 \%$. Finally, the False Negatives average around $0.23 \%$. While this percentage does not seem very high, it is detriment to overall safety since False Negatives cannot be detected and hence, no countermeasures can be taken. It is therefore the focus of this paper to greatly reduce this category.

\section{B. Baseline Vulnerabilities to False Negatives}

In layman terms, the False Negatives using TEC are caused by repetitiveness within the valid code words. Fig. 5 reveals code words with a clear repetition that cause False Negatives: code words consisting of one value, i.e. all-zero and all-one code words, and code words consisting of alternating values, e.g. "...0101...". Even code words where groups of zeroes 


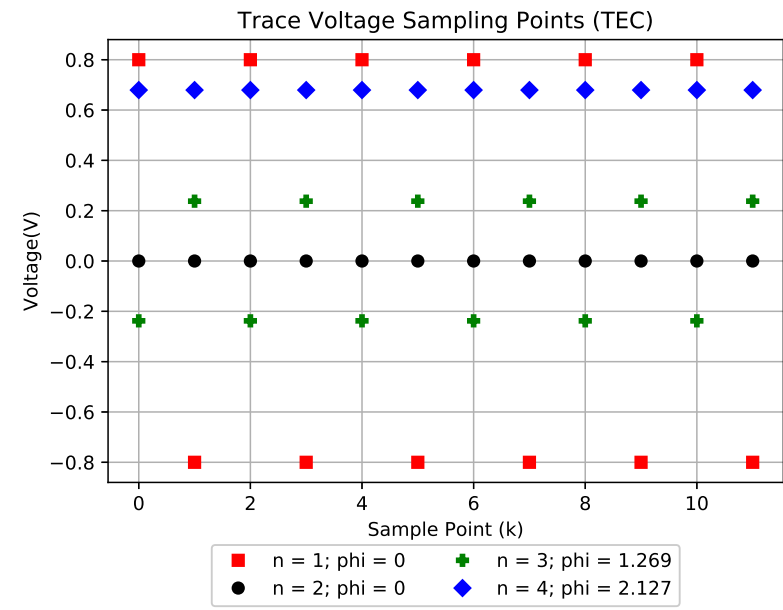

Fig. 5. Sampling Point Periodicity and Value

or groups of ones are alternating, e.g. "...0011001100...", can easily cause a False Negative under EMI disturbances. From [4], it is known that all code words with any repetitive characterestic can cause False Negatives.

Analyzing the data gathered during the study presented in [4] showed that two encoding mechanisms create repetition within a code word: bit order encoding (spatial symmetry) and bit level encoding (code symmetry).

The bit order encoding determines where the triplicated bits are placed within the code word. For example, the bit order encoding used within TEC is to place all three triplicated bits next to each other which creates a high level of repetition. By changing this mechanism, by placing the triplicated bits further apart, a change in EMI-resilience is obtained.

The bit level encoding mechanism determines which value, i.e. a one or a zero, is used to triplicate a given data bit. For instance, the bit level encoding mechanism of TEC is to use the same level for all three bits, mapping a data bit ' 1 ' to '111'. Such bit level encoding mechanism again creates repetition within a code word. Therefore, using a different bit level encoding mechanism enhances the EMI-resilience of the EDCC code.

\section{Simulated EMI Fault Model}

To improve the EMI resilience of the triplication EDCC, three new variants are developed. These variants change one or more encoding mechanisms, identified in Section III-B, in order to decrease the repetitiveness of the code words and thus to improve the EMI-resilience of the code word. First, the new variants are introduced. Then they are submitted to the same EMI-disturbaces used in [4] to allow an equal measure and comparison of their performance. Finally, a systematic fault injection model is used on all four considered triplication variants to further measure their resilience against bit-flips. This systematic fault model is described in Section V.

\section{A. EMI-Resilient EDCCs}

Three new triplication variants are developed to improve the EMI-resilience of the code words:
- The first new triplication variant is denoted as TAN: Triplication with Altered bit order with Nominal encoding (i.e. without inversion). Compared to TEC, this variant only changes the bit order. The encoding scheme is provided in Eq. 7.

$$
T A N(A B C D)=[A B C D C A D B D C B A]
$$

- The second developed variant is called TCILT; Triplication placing the bits Close together with Inversion on the Last Triplet. This encoding changes the bit level encoding by inverting the final bit of the triplicated bits, but has the same bit order encoding as in TEC. The encoding scheme is provided in Eq. 8.

$$
T C I L T(A B C D)=[A A \bar{A} B B \bar{B} C C \bar{C} D D \bar{D}]
$$

- The final considered variant is called TDILT; Triplication with Dispersed data bits with Inversion on the Last Triplet. Compared to TEC, TDILT both have a different bit order encoding mechanism and a different bit level encoding mechanism. The encoding scheme for the final variant is provided in Eq. 9.

$$
T D I L T(A B C D)=[A B C D A B C D \bar{A} \bar{B} \bar{C} \bar{D}]
$$

\section{B. Influence of the Bit Order}

Earlier attempts to reduce the rate of False Negatives by changing the bit order were unsuccessful [4]. Two of the triplication variants used bit interleaving, but our simulations revealed an increased susceptibility to EMI, mainly due to the equal reciprocal distances between the bits. The reciprocal distance between the data bits is the amount of bits between each occurrence of a specific encoded data bit (e.g. 'A'). A different approach, presented in this paper, will make sure that none of the three bits within a data triplet have the same distance. The code introduced as TAN (Section IV-A) is considered, which has an unequal reciprocal inter-bit distance. The resulting fault categories and their distribution are displayed in Fig. 6.

By changing up the order of the bit interleaving, part of the False Negatives have disappeared and the assumption of the responsible factor has proven correct. The rate of False Negatives has decreased by roughly a factor eight, resulting in an overall mean False Negative rate of $0.028 \%$ as compared to the previous value of $0.23 \%$. When observing Fig. 6, there are still False Negatives around the core frequencies denoted earlier in Eq. 3. Whilst reducing the pattern of False Negatives overall, one demand is still unfulfilled. The TAN encoding contains a valid all-one and all-zero code word. This should be resolved by introducing inversion.

One can state that the effectiveness of switching the bit order is good, but not optimal. Whilst reducing the rate of False Negatives, an increase of about $0.1 \%$ is noted within the orange (failed correction) category. This increased is centered around the bit rates denoted in Eq. 3. Therefore it is justified to state that the code is better in terms of False Negatives, but does not provide us with the increased performance and availability we had in mind. 


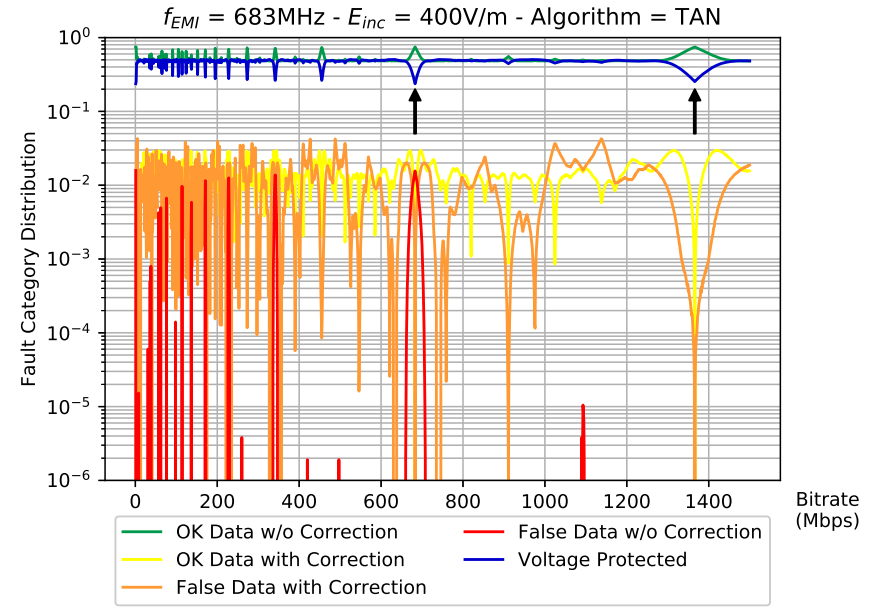

Fig. 6. TAN Fault Categorization Distribution

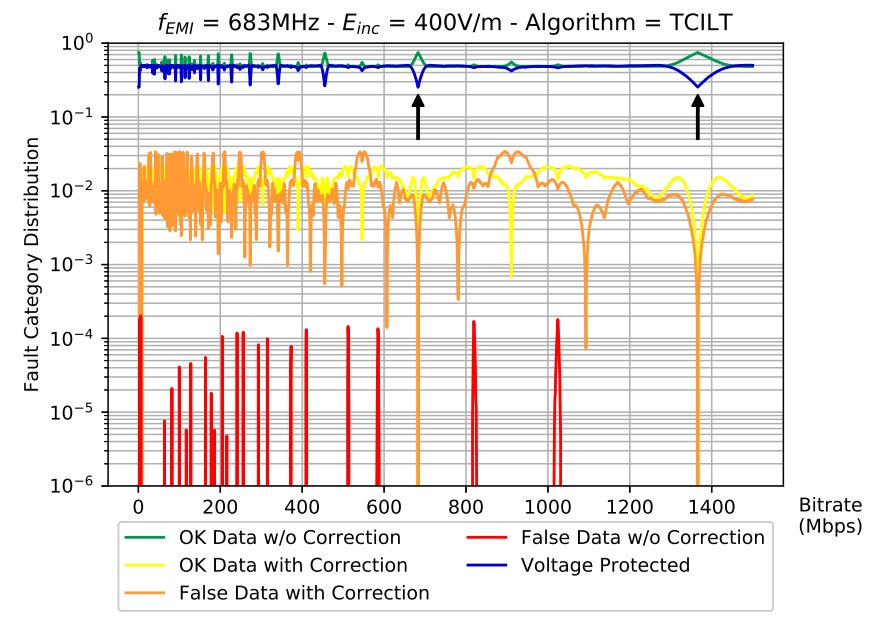

Fig. 7. TCILT Fault Categorization Distribution

\section{Influence of Inversion}

Section IV-B made it clear that only changing the bit order is insufficient. In order to change the code symmetries, we must use inversion. By strategically inverting the bits within the code word, an asymmetric code can be obtained. Previous work [4] already uncovered that introducing inversion does not guarantee a reduction in the rate of False Negatives, where both codes with inversion actually performed worse than the simple triplication TEC. This was because the middle bit of each triplet was inverted, which resulted in a symmetric code. If $A=$ ' 0 ', then the code contained a " 010 ", resulting in an increased susceptibility to EMI. Both newly introduced codes TCILT and TDILT (Section IV-A) resolve this issue.

The first version to be referenced against the baseline is TCILT. The results for these simulations are presented in Fig. 7. At first glance, all categories, except for the False Negatives, are quite similar to TAN, with a slight deviation within the orange (failed correction) category, it is also quite similar to TEC. The overall performance metrics for all codes are mentioned in Table I.

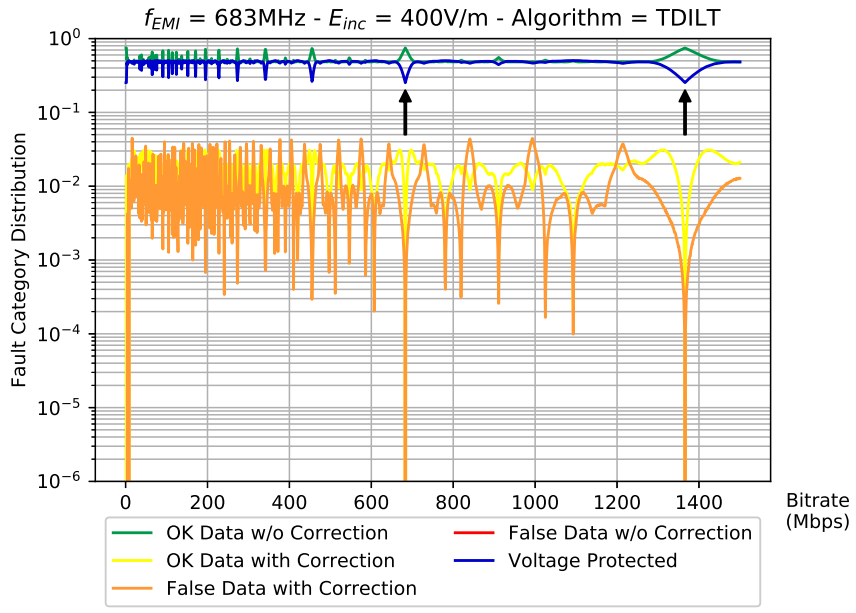

Fig. 8. TDILT Fault Categorization Distribution

TABLE I

Numeric Results Per Category Per Code

\begin{tabular}{ccccc} 
& TEC & TAN & TCILT & TDILT \\
\hline Blue & $4.66 \mathrm{E}-1$ & $4.67 \mathrm{E}-1$ & $4.68 \mathrm{E}-1$ & $4.66 \mathrm{E}-1$ \\
Green & $5.06 \mathrm{E}-1$ & $5.06 \mathrm{E}-1$ & $5.06 \mathrm{E}-1$ & $5.06 \mathrm{E}-1$ \\
Yellow & $1.44 \mathrm{E}-2$ & $1.48 \mathrm{E}-2$ & $1.44 \mathrm{E}-2$ & $1.71 \mathrm{E}-2$ \\
Orange & $1.05 \mathrm{E}-2$ & $1.13 \mathrm{E}-2$ & $1.12 \mathrm{E}-2$ & $1.02 \mathrm{E}-2$ \\
Red & $2.28 \mathrm{E}-3$ & $2.84 \mathrm{E}-4$ & $2.52 \mathrm{E}-6$ & 0.00
\end{tabular}

The major difference is present in the False Negatives category. The False Negatives around the integer multiple bit rates, in reference with Eq. 3, have been greatly reduced. On Fig. 7, we can see that there are still some vulnerabilities left. However, the occurrence of False Negatives has already significantly dropped in magnitude in comparison to TAN on Fig. 6. The rate of False Negatives has dropped to $2.52 * 10^{-6}$. That is almost a 1000 times lower than the baseline TEC. Nonetheless, the remaining False Negatives are (again) caused by repetitiveness. Two data words are causing False Negatives, and from the encoding provided in Eq. 10 should be clear why:

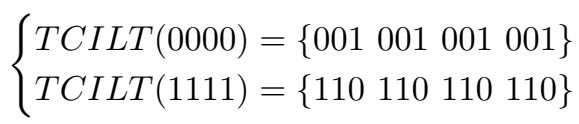

At $1024 \mathrm{Mbps}$, the ratio between the bit rate and disturbance frequency is about 1.5; which leads, combined with the encoding as described in Eq. 10 to a False Negative. By changing the order (even in a systematic way) and inverting the last triplet, this repetitiveness is also eliminated. The reordered encoding scheme has been labeled TDILT and was already shown in Eq. 9. The simulation results for TDILT are shown in Fig. 8, with the accompanying numerical data in Table I.

All observations, and differences in numerical data, are caused by the EMI-diverse techniques implemented by the different codes. For TDILT, the following observations are made:

- The blue (voltage protected) category averages out at $46.6 \%$. Since there is no difference in code length, this category is the same for all considered EDCCs; 
- The green (error-free) category averages around $50 \%$. Thus, in $50 \%$ of the simulated cases, an error-free code word was received. This category is largely dependent on the disturbance frequency and incoming field strength;

- The yellow (sucessful correction) category is the largest of the considered techniques, averaging $1.71 \%$. This $1.71 \%$ is the percentage of corrected code words in total. However, another metric is the percentage of recoverable code words, which were corrupted but not caught by the over-voltage protection system. This metric is the ratio of the yellow category and the sum of the yellow and orange categories. Now the receiver is able to recover $62.7 \%$ of the corrupted code words which did not trigger the over-voltage protection;

- The orange (failed correction) category is the lowest of the considered techniques, averaging $1.02 \%$. This is desired, since orange should be as low as possible, although this category tolerable by the system. The same rationale should be used as with the yellow category: $37.3 \%$ of the corrupted code words (not caught by the over-voltage protection system) are corrected to an incorrect code word;

- The rate of False Negatives has dropped to an absolute zero, under the considered electromagnetic variables, as was the goal and focus of this paper.

At this point, it is justified to state that TDILT is the most robust code against EMI-induced errors within a communication channel, for the considered electromagnetic environment. The authors are aware that False Negatives might still be induced under other, more complex electromagnetic disturbances. Nonetheless, it is expected that TDILT will outperform other triplication variants in terms of the rate of False Negatives.

\section{Systematic Fault Injection Model}

The findings in Sections III and IV already showed that False Negatives occur within the considered codes, with the exception of TDILT. However, more complex electromagnetic disturbances might still induce False Negatives in the TDILT variant. Since this paper focuses on False Negatives, we investigate the inherent rate of False Negatives within all considered triplication variants.

To characterize the inherent rate of False Negatives, all possible errors for a given code were injected in a systematic way. By working this way, we can assess if a decrease in the rate of False Negatives is due to the specific consideration of the EMI disturbance, or a decrease in the inherent rate of the code.

The main difference with the EMI simulations is that all possible errors occur here with the same probability, which enables the assessment of its performance, regardless of the considered electromagnetic environment. It also allows to find all possible corruptions of the code words that might lead to False Negatives. In turn, this knowledge is used to increase the resilience of the code. In essence, this theoretical fault model allows to see which code has the lowest inherent False Negative ratio, and allows to determine possible alterations to limit the impact of EMI.

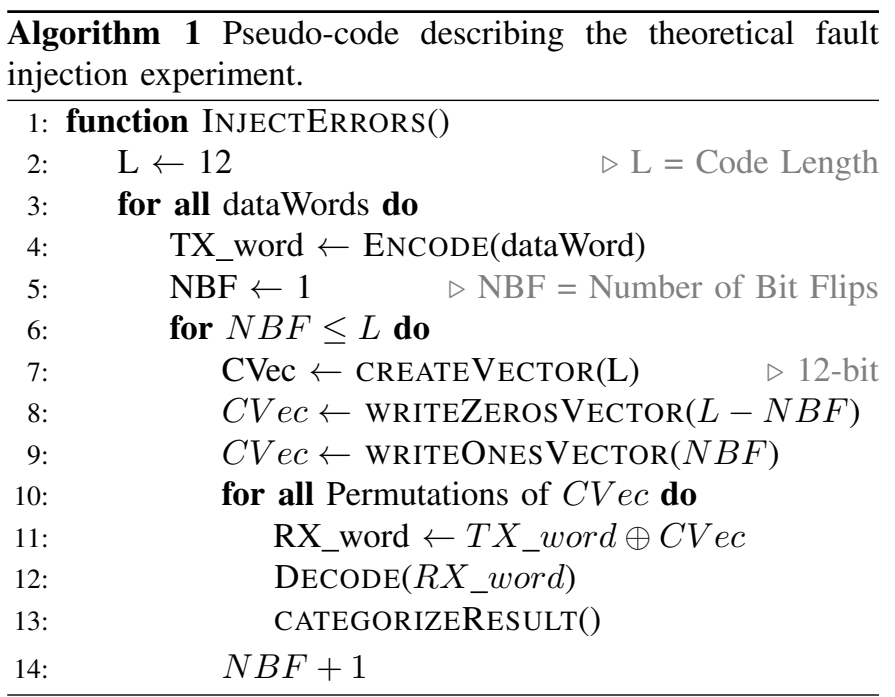

\section{A. Fault Injection Algorithm}

To create the theoretical baseline, a systematic approach is taken to inject all possible bit errors for a certain given code length $L$. This systematic approach is described in Algorithm 1. To insert errors, or bit flips, the easiest way is to XOR the value of the code word with a logical ' 1 '. The XOR function transforms a ' 0 ' into a ' 1 ' and vice versa. This is given by Eq. 11 .

$$
\left\{\begin{array}{l}
0 \oplus 1=1 \\
1 \oplus 1=0
\end{array}\right.
$$

In essence, all possible 12-bit bit streams (the corrupting vector) are XOR-ed with the originally encoded data; thus $2^{12}$ or 4096 injections in total per data word. Algorithm 1 shows that all possible 4-bit data words are considered (Line 2). Each of the data words is encoded and the loop control variable (Number of Bit Flips or $N B F$ ) is initialized. For each of the $N B F$, the following is performed:

1) Create a corruption vector $(C V e c)$. This vector holds twelve values in total. This is equal to the considered code length $L$. It is filled with $L-N B F$ zeros and $N B F$ ones. From Eq. 11 is known that each ' 1 ' inserts a bit flip. For the first loop, a single-bit bit-flip is injected. For the second loop, two-bit bit-flips are injected, etc. Within Algorithm 1, this step is Line 7-9.

2) The first permutation (Line 10) to be considered, is the layout as described in the above step. The first permutation injects the faults all the way at the back of the code word. It was specified as such, because the permutations are done in lexicographical ascending order. This again gives a systematic approach, leading to full coverage of the injected errors. By using the lexicographical order, we make sure that each permutation is unique. As an example, a 4-bit corruption vector is used with two bit flips to be injected. The first permutation is thus $[0,0,1,1]$. This is also the smallest binary value. The next permutation are, in specified order: $[0,1,0,1]$, $[0,1,1,0],[1,0,0,1],[1,0,1,0]$ and $[1,1,0,0]$. There are thus 


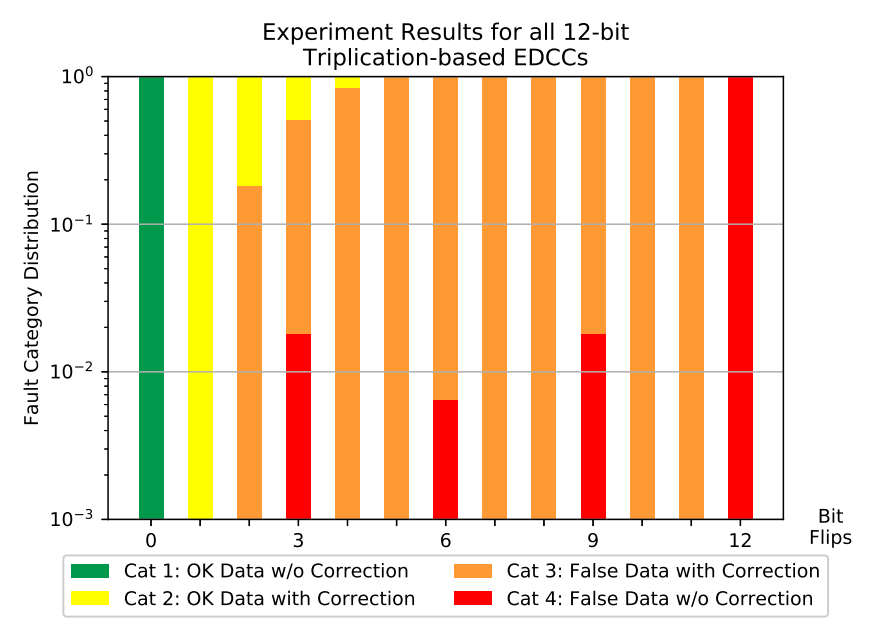

Fig. 9. Fault Injection Experiment Results for Triplication-based EDCCs

6 distinct permuations, which can be calculated as shown in Eq. 12.

$$
\text { \#Permutations }=\frac{n !}{k !(n-k) !}=\frac{4 !}{2 ! 2 !}
$$

3) For each permutation, the bit flips are injected. By performing the XOR operation between the corruption vector and the originally encoded data, the received code word with errors is created. This received code word is then decoded and the result is categorized into the categories mentioned in Section II-C. The fifth category (over-voltage protected) is considered, but not shown. The systematic fault injection model considers all possible bit flips, which are not detected by the overvoltage mechanism.

\section{B. Fault Model Outcomes}

The results of this fault injection experiment are the same for all codes and are presented in Fig. 9. Note that the trivial case of zero bit flips (always correct) and $L$ bit flips (always a False Negative) are also presented within the figure.

For all permutations of a single bit flip, we see that the code is able to recover. This is expected behavior, as triplication is a fault masking technique and a single fault is perfectly recovered by the majority voting mechanisms. For more than a single bit flip, the capabilities of recovering the errors depend on the place they happened. For example, most of the time, a two-bit bit flip can be recovered. However, Fig. 9 shows an orange category (corrected to an invalid code word) for around $18 \%$ of the two-bit bit flips. This occurs when both faults are injected into the same triplet, causing an erroneous majority vote. Of course, the more bit flips, the more faults are injected into the same triplet. This is reflected by the gradual decrease of the amount of succesful corrections (yellow) and being replaced with failed corrections (orange).

Obviously, this experiment goes well beyond the design limits of the codes under consideration. All triplication-based codes are Single Error Correction (SEC) codes. They can
TABLE II

FAULT INJECTION EXPERIMENTS - NUMERICAL RESULTS (IN \%)

\begin{tabular}{c|cc|c|ccc} 
NBF & Yellow & Orange & NBF & Yellow & Orange & Red \\
\hline 1 & 100.00 & 0.00 & 2 & 81.82 & 18.18 & 0.00 \\
5 & 0.00 & 100.00 & 3 & 49.09 & 49.09 & 01.82 \\
7 & 0.00 & 100.00 & 4 & 14.36 & 83.64 & 0.00 \\
8 & 0.00 & 100.00 & 6 & 0.00 & 99.35 & 00.65 \\
10 & 0.00 & 100.00 & 9 & 0.00 & 98.18 & 01.82 \\
11 & 0.00 & 100.00 & & & &
\end{tabular}

TABLE III

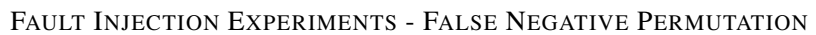
NUMBER

\begin{tabular}{cccc} 
\# NBF & \# Permutations & \# FNs & Ratio \\
\hline 3 & 220 & 4 & $1.82 \%$ \\
6 & 924 & 6 & $0.65 \%$ \\
9 & 220 & 4 & $1.82 \%$ \\
12 & 1 & 1 & $100 \%$
\end{tabular}

correct (or mask) a single error within the code word. This statement goes without any other conditions. Once multi-bit bit flips are considered, conditions such as the faults being injected in separate triplets, emerge. Therefore, the design limit is single error correction only. When surpassing the design limits, especially with EMI, the main focus is the occurrence of False Negatives.

The exact numbers making up Fig. 9 are listed in Table II. The left side of Table II shows for which amount of bit flips (NBF), there is only one kind of error. The right side shows the number of bit flips where, depending on where in the triplets the faults are injected, more fault categories are possible.

As can be seen from Fig. 9 and Table II, False Negatives only arise when the number of bit flips is a multiple of three. This is logical, since three bits of a triplet need to be corrupted before a False Negative can arise. The corruption of one, two, three or four triplets, will therefore take three, six, nine or twelve bit flips respectively. The percentage noted in Table II are deducted from the ratio between the amount of False Negative inducing permutations and the total possible permutations for a given number of bit flips $(N B F)$. Thus, multiplying the ratio by the total permutations for a given $N B F$ gives the False Negative inducing permutations (Eq. 13):

$$
\begin{cases}F N(3)=1.82 \% \rightarrow 0.0182 \times \frac{L !}{3 !(L-3) !} & =4 \\ F N(6)=0.65 \% \rightarrow 0.0065 \times \frac{L !}{6 !(L-6) !} & =6 \\ F N(9)=1.82 \% \rightarrow 0.0182 \times \frac{L !}{9 !(L-9) !} & =4 \\ F N(12)=100 \% \rightarrow 1 \times \frac{L}{12 !(L-12) !} & =1\end{cases}
$$

From Eq. 13 it is clear that only a handful of corruptions lead to False Negatives. For a three-bit bit flip, that is only 4 out of 220 permutations. For a six-bit bit flip, that is 6 out of 924 possible permutations. The nine-bit bit flip equals the three-bit bit flip with 4 out of 220 permutations and the twelve-bit corruption is a trivial case with only one possible permutation. This is also shown in Table III for clarity. Table III clearly shows that even though a lower percentage is observed, this is due to the higher amount of permutations that exist for six-bit bit flips. 
The phenomena witnessed by the outcome of this theoretical fault model are called homopolar faults (derived from homopolar currents in [13]). Homopolar faults arise when the disturbance is in phase with the original frequency. Since the codes are based on triplication, the multiples of three bit-flips will be the cause for False Negatives. The values above denote this phenomenon: three-, six-, nine- and twelve-bit bit flips cause the False Negatives.

Important to realize is that this fault injection model and its False Negatives, is only the fourth step when surveying a system under harsh EM disturbances. Actually, four conditions must be met for a False Negative to arise:

1) The over-voltage protection system should not be triggered; i.e. the field strength should not be too large, or the frequency must be just right according to Eq. 3;

2) The code word must be corrupted; i.e. the incoming field strength should be sufficiently large;

3) The corrupted code word must contain either three, six, nine or twelve bit flips;

4) The placement of the corrupted bits must be according to one of the possible False Negative inducing permutations, as noted in Eq. 13.

As far as the systematic fault injection model is concerned, there is no difference in using EMI-resilience techniques. This is because this model does not consider any statistical likelihood of errors to be induced. The likelihood of a specific corruption leading to a False Negative is solely dependent on the environment the device is operating in. The likelihood for a False Negative to arise within the TEC variant, are far larger than the likelihood of a False Negative occurring within TDILT. From the systematic fault injection, there are still possibilities to disrupt TDILT and get False Negatives.

This is the specific added value of covering the entire spacestate of the possible faults: the perfect code does not exist, but it is possible to limit the rate of False Negatives under continuous wave EMI.

\section{CONCLUSIONS}

False Negatives, where corrupted data is deemed valid, are detrimental for overall system safety and performance. Since these errors cannot be detected, countermeasures cannot be taken. In light of harsh continuous-wave electromagnetic disturbances, this paper presented different resilience techniques which can be implemented in currently used EDCCs. These techniques for improved EMI-resilience were tested with a Monte-Carlo-based continuous wave EMI simulation framework.

Alongside the EMI simulations, the performance of the codes was assessed with systematic fault injection experiments. It is found that all triplication-based variants have exactly the same performance under the considered fault model. However, when these variants are exposed to a continuouswave EMI disturbance, there are major differences in performance and, as the focus of this paper, in the rate of False Negatives.

This paper succeeds, by strategically using inversion, in eliminating the False Negatives from the simulations, effectively creating a more EMI resilient code. Even though this code might still generate these False Negatives under other, more complex electromagnetic phenomena, the authors are convinced that the EM-resilient variant will perform better, except for perhaps very specific disturbances.

Besides eliminating the False Negatives, we also succeeded in lowering the amount of wrong corrections; i.e. a correction was performed by the code, but it is not equal to the originally transmitted data. This leaves a higher percentage of data that is either error-free, covered by the over-voltage protection system or corrected. As such, the overall safety and availability of a system operating in harsh environments is increased. Of course, the imposed overhead on the communication channel must be tolerable by the system.

Overall, when working in harsh environments, a code constructed with the right inversion and bit interleaving, will increase the dependability of a communication channel.

\section{REFERENCES}

[1] IEC 61508: Functional safety of electrical/electronic/programmable electronic safety-related systems, International Electrotechnical Commission (IEC) Std., 2010.

[2] D. Pissoort and A. Degraeve and K. Armstrong, "EMI Risk Management: A necessity for safe and reliable electronic systems!" in Proc. IEEE 5th Int. Conf. Consumer Electronics - Berlin (ICCE-Berlin), Sep. 2015, pp. 208-210.

[3] D. Pissoort and K. Armstrong, "Why is the IEEE developing a standard on managing risks due to EM disturbances?" in 2016 IEEE International Symposium on Electromagnetic Compatibility (EMC), July 2016, pp. 7883.

[4] J. Van Waes, J. Lannoo, J. Vankeirsbilck, D. Vanoost, D. Pissoort, and J. Boydens, "Resilience of error correction codes against harsh electromagnetic disturbances: Fault mechanisms," IEEE Transactions on Electromagnetic Compatibility, 2019, early Access.

[5] J. Lannoo, J. Van Waes, A. Degraeve, D. Vanoost, J. Boydens, and D. Pissoort, "Effectiveness of time diversity to obtain EMI-diverse redundant systems," in Proc. Int. Symp. Electromagnetic Compatibility (EMC EUROPE), Aug. 2018, pp. 288-292.

[6] J. Van Waes, J. Lannoo, A. Degraeve, D. Vanoost, D. Pissoort, and J. Boydens, "Effectiveness of cyclic redundancy checks under harsh electromagnetic disturbances," in Proc. Int. Symp. Electromagnetic Compatibility - EMC EUROPE, Sep. 2017.

[7] F. Vanhee, D. Pissoort, J. Catrysse, G. A. E. Vandenbosch, and G. G. E. Gielen, "Efficient reciprocity-based algorithm to predict worst case induced disturbances on multiconductor transmission lines due to incoming plane waves," IEEE Transactions on Electromagnetic Compatibility, vol. 55, no. 1, pp. 208-216, Feb. 2013.

[8] D. A. Hill, "Plane wave integral representation for fields in reverberation chambers," IEEE Transactions on Electromagnetic Compatibility, vol. 40, no. 3, pp. 209-217, Aug. 1998.

[9] M. Magdowski, S. V. Tkachenko, and R. Vick, "Coupling of stochastic electromagnetic fields to a transmission line in a reverberation chamber,' IEEE Transactions on Electromagnetic Compatibility, vol. 53, no. 2, pp. 308-317, May 2011.

[10] J. Shi, "Esd characteristics of ggnmos device in deep sub-micron cmos technology," 2016 International Conference on Audio, Language and Image Processing (ICALIP), pp. 327-331, 2016.

[11] J. Van Waes, J. Lannoo, J. Vankeirsbilck, A. Degraeve, J. Peuteman, D. Vanoost, D. Pissoort, and J. Boydens, "Effectiveness of hamming single error correction codes under harsh electromagnetic disturbances," in Proc. Int. Symp. Electromagnetic Compatibility - EMC EUROPE, 2018.

[12] J. Van Waes, J. Vankeirsbilck, J. Lannoo, D. Pissoort, and J. Boydens, "Effectiveness of data triplication in harsh electromagnetic environments," in Proc. Int. Symp. Electromagnetic Compatibility - EMC EUROPE, 2018.

[13] H. Arghavani and M. Peyravi, "Unbalanced current-based tariff," CIRED-Open Access Proceedings Journal, vol. 2017, no. 1, pp. 883 887, 2017. 